\title{
Curcumin Attenuates Oxaliplatin-Induced Liver Injury and Oxidative Stress by Activating the Nrf2 Pathway
}

This article was published in the following Dove Press journal:

Drug Design, Development and Therapy

\author{
Yulei Lu (1D) \\ Shengming $\mathrm{Wu}^{2}$ \\ Bangde Xiang ${ }^{3}$ \\ Lequn $\mathrm{Li} \mathbb{D}^{3}$ \\ Youzhi $\operatorname{Lin}^{3}$ \\ 'Guangxi Medical University Cancer \\ Hospital, Nanning, Guangxi 53002I, \\ People's Republic of China; \\ ${ }^{2}$ Departments of Pathology, Guangxi \\ Medical University Cancer Hospital, \\ Nanning, Guangxi 53002I, People's \\ Republic of China; ${ }^{3}$ Departments of \\ Hepatobiliary Surgery, Guangxi Medical \\ University Cancer Hospital, Nanning, \\ Guangxi 53002I, People's Republic of \\ China
}

Correspondence: Youzhi Lin Departments of Hepatobiliary Surgery, Guangxi Medical University Cancer Hospital, 7I He Di Road, Nanning, Guangxi Zhuang Autonomous Region 530021 , People's Republic of China Email linyouzhi200I@I63.com
Purpose: Oxaliplatin (OXA)-induced liver injury is one of the main limiting factors affecting the efficacy of OXA-based chemotherapy in patients with colorectal liver metastases. In addition, oxidative stress is an important pathophysiological mechanism of OXAinduced liver injury. Therefore, dietary antioxidants may decrease or prevent hepatic toxicity in vivo and be beneficial to OXA-based chemotherapy.

Methods: An experimental OXA-induced liver injury animal model was established, and the protective effects of curcumin (CUR) against OXA-induced liver injury were investigated. ELISA was used to determine the levels of MDA, SOD, CAT, and GSH in liver tissue. The effect of CUR treatment on the expression of cytokines and the Nrf2 pathway was determined by real-time PCR and Western blotting.

Results: CUR treatment alleviated OXA-induced hepatic pathological damage and splenomegaly. The protective effect of CUR was demonstrated to be correlated with inhibition of oxidative stress, inflammation, and the coagulation system. Furthermore, Western blotting revealed that CUR treatment reverses the suppression of Nrf2 nuclear translocation and increases the expression of HO-1 and NOQ1 in mice with OXA-induced liver injury. Moreover, the Nrf2 activation and hepatoprotective effect of CUR were abolished by brusatol.

Conclusion: Curcumin attenuates oxaliplatin-induced liver injury and oxidative stress by activating the Nrf2 pathway, which suggests that CUR may be potentially used in the prevention and treatment of OXA-induced liver injury.

Keywords: oxaliplatin, curcumin, liver injury, oxidative stress, $\mathrm{Nrf} 2$

\section{Introduction}

The majority of chemotherapeutic drugs are not target-specific and cannot distinguish between tumor cells and normal non-cancerous cells, resulting in toxicity and limiting their clinical use. ${ }^{1}$ Therefore, the identification of effective adjuvant drugs to reduce the toxicity of existing chemotherapeutic drugs and to achieve better antitumor treatment effects is warranted.

Oxaliplatin (OXA), a third-generation platinum chemotherapeutic agent, is widely used in the treatment of several cancers such as colorectal cancer and gastric cancer. ${ }^{2}$ OXA-based chemotherapy for colorectal liver metastases (cRLM) has increased resection rates and improved outcomes, and is therefore recommended as the first-line basic chemotherapeutic drug. ${ }^{2,3}$ However, OXA-induced liver injury is a primary limiting factor of OXA-based chemotherapy in patients with cRLM. ${ }^{4}$ Studies have revealed 
OXA-induced liver injury in patients who underwent preoperative OXA-based chemotherapy, with an incidence rate of $19-78 \%{ }^{5,6}$ Other reports have shown that OXA-induced sinusoidal injury, one of the distinct drug-specific sideeffects of OXA, is associated with intraoperative bleeding and postoperative morbidity, as well as early recurrence and decreased overall survival. ${ }^{7,8}$ The pathological features of OXA-induced liver injury include hepatic sinusoidal dilatation, intrahepatic sinus platelet aggregation, hepatic steatosis, and clinically important adverse effects characterized by a bluish hue in the liver, splenomegaly, and thrombocytopenia. ${ }^{4,9}$ To overcome these side effects, an effective adjuvant drug that protects the liver against damage caused by OXA is imperative.

Previous studies have demonstrated that oxidative stress is one of the major mechanisms implicated in OXA-induced liver injury. ${ }^{10}$ OXA has been shown to generate oxidative damage, and is therefore responsible not only for an increase in lipid peroxidation, but also for reduction in antioxidant glutathione (GSH) levels and inhibition of the activity of various antioxidant enzymes, leading to an imbalance between the oxidative and antioxidative systems, and ultimately liver injury. ${ }^{11}$ In addition, the pathogenesis of OXA-induced liver injury has been associated with the downregulation of genes related to oxidative stress, including metallothionein 1 (Mt1), heme oxygenase 1 (HO1), superoxide dismutase 3 (SOD3), and nuclear factor erythroid 2-related factor 2 (Nrf2). ${ }^{10}$ Accordingly, natural products with potent liver protective effects, such as curcumin and dioscin, have been shown to be effective in the treatment of OXA-induced liver injury. Indeed, several antioxidative compounds can ameliorate OXA-induced liver injury by reducing oxidative stress in a murine model. ${ }^{10,12,13}$ Schwingel et al reported that the antioxidative compounds resveratrol, quercetin, and quercetin nanoemulsion can effectively alleviate OXA-induced liver toxicity in a murine model. ${ }^{14}$ Dietary supplementation with the antioxidant butylated hydroxyanisole (BHA) has been shown to prevent the development of OXA-induced Sinusoidal obstruction syndrome (SOS) in a murine model. ${ }^{10}$

Curcumin (CUR), a hydrophobic polyphenol present in turmeric, has been unequivocally recognized for its hepatoprotective, antioxidant, anti-inflammatory, and anticarcinogenic functions. ${ }^{1,15,16}$ Especially in terms of its hepatoprotective effects, increasing evidence demonstrates that CUR exerts protective effects against toxins such as carbon tetrachloride, ${ }^{17}$ alcohol, ${ }^{18} \mathrm{~N}$-nitrosodiethylamine, ${ }^{19}$ and arsenic, ${ }^{20}$ and these effects are related to its antioxidant activity. ${ }^{21}$ Several studies have suggested that CUR possibly acts through activating the Nrf2 signaling pathway, effectively protecting the liver from oxidative damage induced by toxicants. ${ }^{20,22}$ Additionally, increasing data shows that CUR activates Nrf2, which further initiates the expression of a variety of antioxidant enzymes and Phase II drug-metabolizing enzymes, including HO-1 and NAD(P) H:quinine oxidoreductase 1 (NQO1), and finally protects various tissues and cells against oxidative stress. ${ }^{16,20}$ However, very little is known about the protective effects of CUR against OXA-induced liver injury in vivo. It is still uncertain whether CUR can reverse the typical histopathological alterations induced by OXA and whether the Nrf2 signaling pathway is involved in this process.

In this study, we investigated the protective effects of CUR against OXA-induced liver injury in vivo. To better simulate the pathogenic process of OXA-induced liver injury, an animal model was established, where the drug was administered to animals based on a treatment schedule similar to patients. We also investigated histopathological changes and mechanisms underlying the hepatoprotective effects of CUR against OXA-induced liver injury, including oxidative stress, inflammatory responses, and Nrf2 signaling pathway activation.

\section{Materials and Methods}

\section{Reagents}

OXA was obtained from Hengrui Medicine Co., Ltd. (Jiangsu, China). CUR $\left(\mathrm{C}_{21} \mathrm{H}_{20} \mathrm{O}_{6}, \mathrm{MW}=368.39\right.$, analytical reagent) was purchased from Sinopharm Chemical Reagent Co. (Beijing, China) and dissolved in 1\% sodium carboxymethylcellulose Solutions were freshly prepared on each experimental day. Alanine aminotransferase (ALT), aspartate aminotransferase (AST), reduced glutathione (GSH), superoxide dismutase (SOD), catalase (CAT), malondialdehyde (MDA), and total protein quantification (BCA method) kits were obtained from the Nanjing Jiancheng Bioengineering Institute (Nanjing, China).

\section{In vivo Chemotherapy Model}

To determine the direct toxicity of OXA, BALB/cJ mice (age: 6-8 weeks) purchased from Beijing Vital River Laboratory Animal Technology Co., Ltd. (Beijing, China) were used. All mice were housed under standardized conditions with five mice in each cage. The mice had ad libitum access to standard chow and water and one week to adapt to the laboratory environment prior to manipulation. The 
cages were placed in a room with a temperature of $22-25^{\circ} \mathrm{C}$, 45-55\% humidity, and a 12-h light-dark diurnal cycle (lights on between 7:00 AM and 7:00 PM).

To establish the in vivo chemotherapy model, the $\mathrm{BALB} / \mathrm{cJ}$ mice were randomly divided into two groups ( $\mathrm{n}=18$ per group), (1) control group: The mice were treated with a vehicle $(5 \%$ glucose $0.5 \mathrm{~mL})$ on a weekly basis for eight weeks; (2) OXA group: The mice were treated with $8 \mathrm{mg} / \mathrm{kg}$ OXA $(0.5 \mathrm{~mL})$, which was administered via intraperitoneal injection (i.p.) on a weekly basis for a total of eight weeks. The drug dosing schedule was based on previously published studies and our preliminary experiments. $^{10,12}$ To investigate whether OXA induced liver injury and splenomegaly in mice, mice $(n=6$ per group) were euthanized one week after the fourth, sixth and eighth administration of OXA under isoflurane anesthesia by cardiac puncture, and then blood, liver tissues and spleens were collected for further analysis.

To investigate the protective effect of CUR, the BALB/cJ mice were randomly divided into five groups $(n=5$ per group): (1) control group: mice were treated with 5\% glucose (0.5 mL) on a weekly basis for eight weeks; (2) CUR group: mice were treated with $100 \mathrm{mg} / \mathrm{kg}$ CUR via gavage for eight weeks; (3) OXA group: mice were treated with $8 \mathrm{mg} / \mathrm{kg}$ OXA (i.p.) on a weekly basis for eight weeks; (4) OXA $+50 \mathrm{mg} / \mathrm{kg}$ CUR group: mice were treated with $8 \mathrm{mg} / \mathrm{kg}$ OXA (i.p.) on a weekly basis plus CUR $(50 \mathrm{mg} / \mathrm{kg}$ via gavage $30 \mathrm{~min}$ before and three days after each OXA injection) for eight weeks; and (5) OXA $+100 \mathrm{mg} / \mathrm{kg}$ CUR group: mice were treated with $8 \mathrm{mg} / \mathrm{kg}$ OXA (i.p.) on a weekly basis plus CUR (100 mg/kg via gavage $30 \mathrm{~min}$ before and three days after each OXA injection) for eight weeks. The doses for CUR were selected on the basis of previously published studies. ${ }^{19,20,23}$ The mice were euthanized one week after the final dose of OXA under isoflurane anesthesia by cardiac puncture, and then blood and liver tissues were collected for further analysis.

To investigate the mechanism of CUR, $25 \mathrm{BALB} / \mathrm{cJ}$ mice were randomly divided into five groups ( $\mathrm{n}=5$ per group), (1) control group: mice were treated with vehicle; (2) Brusatol group: brusatol $(2 \mu \mathrm{mol} \cdot \mathrm{kg}-1)$ was administered via i.p. on a weekly basis for eight weeks; (3) OXA group: mice were treated with $8 \mathrm{mg} / \mathrm{kg}$ OXA (i.p.) on a weekly basis for eight weeks; (4) OXA+CUR group: mice were treated with $8 \mathrm{mg} /$ kg OXA (i.p.) on a weekly basis plus CUR (100 mg/kg via gavage $30 \mathrm{~min}$ before and three days after OXA injection) for eight weeks; and (5) OXA+ CUR+ Bru group: mice were treated with $8 \mathrm{mg} / \mathrm{kg}$ OXA (i.p.) on a weekly basis plus CUR
(100 mg/kg, gavage, respectively for $30 \mathrm{~min}$ before and three days after each OXA injection) for eight weeks, and brusatol ( $2 \mu \mathrm{mol} \cdot \mathrm{kg}-1$, i.p.) was administered $2 \mathrm{~h}$ before each CUR gavage. Brusatol (purity 98\%, molecular weight: 520.5) purchased from Adamas Reagent Co., Ltd. (Shanghai, China) was dissolved in normal saline. The mice were euthanized one week after the final dose of OXA, and liver tissues were collected for Western blot analysis.

\section{Determination of MDA, SOD, CAT, and GSH Levels}

The livers from each mouse were homogenized on ice with $5 \mathrm{~mL}$ of $5 \%$ trichloroacetic acid (TCA) per gram of tissue weight. The homogenates were centrifuged at $1000 \mathrm{~g}$ for $15 \mathrm{~min}$ at $4^{\circ} \mathrm{C}$ and the supernatant was collected for further biochemical analysis. The levels of MDA, SOD, CAT, and GSH were determined using commercial reagent kits based on the manufacturers' recommendations.

\section{Real-Time Quantitative PCR}

Total RNA was extracted from liver tissue using TRIzol (Thermo Fisher Scientific, Inc.) and reverse transcription (RT) was performed with the PrimeScript RT reagent kit, according to the manufacturer's instructions (Cat. No. RR036A; Takara Bio, Inc., Otsu, Japan). QPCR was run on a Bio-Rad iQ5 optical module (Bio-Rad Laboratories, Inc., Hercules, CA, USA). Specific primer pairs for different genes were designed as follows: chemokine ( $\mathrm{C}-\mathrm{X}-\mathrm{C}$ motif) ligand 1 (CXCL1), forward 5'-GGCTGGGATTCACCTCAAG-3' and reverse 5'-GCGACCATTCTTGAGTGTG-3'; chemokine (C$\mathrm{X}-\mathrm{C}$ motif) ligand 2 (CXCL2), forward 5'-CCAACCAC CAGGCTACAGG-3' and reverse 5'-GCGTCACACTCA AGCTCTG-3'; monocyte chemotactic protein-1 (MCP-1), forward 5'-AGGTCCCTGTCATGCTTCTG-3' and reverse 5'-TCTGGACCCATTCCTTCTTG-3'; plasminogen activator inhibitor 1 (PAI-1), forward 5'-GATGCTATGGGATTCAAA GTCA-3' and reverse 5'-TCCACCTGTTTCACCATAGTCT -3'; and von Willebrand factor (vWF), forward 5'-CGGGAA GAGTGTGATGGTTGAC-3' and reverse 5'-AGCATCTCCC ACAGCATTCACC-3'. ${ }^{10}$

\section{Western Blotting Analysis}

For Western blotting, liver proteins were extracted. Briefly, total proteins were isolated from the liver tissues using a protein extraction kit (Beyotime Biotechnology, Shanghai, China), and nuclear proteins were isolated using a nuclear and cytoplasmic protein extraction kit (Beyotime Biotechnology, 
Shanghai, China) according to manufacturer's recommendations. Protein concentrations were determined using the BCA protein assay kit (Beyotime Biotechnology, Shanghai, China). Equal amounts of proteins were loaded onto an SDSpolyacrylamide gel for electrophoresis and then transferred onto a polyvinylidene-difluoride (PVDF) membrane using a transblotting apparatus. The blots were immersed in blocking solutions (PBS containing $80 \mathrm{mmol} / \mathrm{L} \mathrm{Na} \mathrm{HPO}_{4}, 25$ $\mathrm{mmol} / \mathrm{L} \mathrm{NaH} \mathrm{PO}_{4} \cdot 2 \mathrm{H}_{2} \mathrm{O}, 100 \mathrm{mmol} / \mathrm{L} \mathrm{NaCl}, 0.1 \%$ Tween20 , and $5 \%$ skim milk) for $1 \mathrm{~h}$ at room temperature. The blots were then incubated with the primary antibodies of anti-Nrf2 (1:500), anti-HO-1 (1:1000), anti-NQO1 (1:1000), anti-lamin B2, and anti- $\beta$-actin (1:1000) (Santa Cruz Biotechnology, CA, USA) at $4^{\circ} \mathrm{C}$ overnight. The next day, the membranes were washed and incubated with the corresponding secondary antibodies (1:1000-5000 dilution) for $2 \mathrm{~h}$ at room temperature. Protein bands were detected using an enhanced chemiluminescence Western blot detection kit. The intensity of each band was quantified using Image Pro Plus 6.0.

\section{Statistical Analysis}

All data were expressed as the mean \pm standard deviation. The data were analyzed using SPSS 19.0 by one-way ANOVA followed by Tukey's post-hoc test or unpaired Student's $t$-test. Differences with $P<0.05$ were considered statistically significant.

\section{Results}

\section{Development of a Reproducible in vivo Mouse Model for OXA-Induced Liver Injury and Splenomegaly}

To establish a mouse model for OXA-induced liver injury, BALB/cJ mice were treated with OXA (i.p.) for eight weeks. The mice exhibited reduced appetite and mild diarrhea after OXA treatment. Body weight gain increased over time in the control group, whereas those of the OXA group were significantly lower, starting two weeks after the first OXA injection and continuing to decrease with further injections $(P<0.05)$ (Figure 1A). No animal died during the experiment. The presence of hepatotoxicity was associated with evidence of hepatocellular injury, as demonstrated by elevated AST levels $(P<0.05)$, but not ALT levels $(P>0.05)$ in the OXA treatment group (Figure 1B). A blinded review of $\mathrm{H} \& \mathrm{E}$ stained sections of the liver demonstrated evidence of sinusoidal dilatation and hepatocyte injury in the OXA-treated mice but not in the control arm (Figure 1C). Besides sinusoidal dilatation and hepatocyte injury, other histological changes, such as steatosis, and inflammatory cell infiltration, were also observed in the OXA group (Figure 1D). The edge of the spleen had a saw-tooth profile after treatment with OXA for four weeks, mild splenomegaly and the saw-tooth edge were observed at six weeks, and splenomegaly was detected after eight weeks of OXA treatment $(P<0.01)$ (Figure $1 \mathrm{E}$ and $\mathrm{F}$ ). This experiment was repeated on 3 separate occasions, and the histological changes of liver and splenomegaly were found to be entirely reproducible with minimal variation in the severity of injury between repeats.

\section{CUR Attenuates OXA-Induced Liver Injury and Splenomegaly}

The administration of CUR (50 mg/ $\mathrm{kg}$ and $100 \mathrm{mg} / \mathrm{kg}$, via gavage) commenced $30 \mathrm{~min}$ before and three days after each OXA injection. H\&E-stained liver sections were blindly reviewed by a pathologist and graded according to the degree of histological damage. Obvious sinus dilatation, steatosis, and inflammatory cell infiltration were observed in the OXA group (Figure 2A). Both 50 and $100 \mathrm{mg} / \mathrm{kg}$ CUR treatment significantly attenuated the pathological sinusoidal injury damage in the liver of the experimental animals. Serum AST activity in the OXA group significantly increased after OXA administration compared to the normal group, while CUR treatment could reverse that increase effectively, as evidenced by AST activity that was markedly increased with $50 \mathrm{mg} / \mathrm{kg}$ and $100 \mathrm{mg} / \mathrm{kg}$ CUR treatment $(P<0.01)$ (Figure 2B). Moreover, CUR administration $(50 \mathrm{mg} / \mathrm{kg}$ and $100 \mathrm{mg} / \mathrm{kg}$ ) attenuated the OXA-induced splenomegaly in mice $(P<0.01)$ (Figure $2 \mathrm{C}$ and $\mathrm{D})$. These results indicated that CUR attenuates OXA-induced liver injury and splenomegaly in mice.

\section{CUR Attenuates OXA-Induced Oxidative Stress}

Treatment with OXA was associated with oxidative stress in the liver, as indicated by an increase in MDA levels $(P<0.05)$ (Figure 3A). Administration of CUR $(50 \mathrm{mg} / \mathrm{kg}$ and $100 \mathrm{mg} / \mathrm{kg}$ ) significantly decreased the MDA levels compared to the OXA group $(P<0.05)$ (Figure $3 \mathrm{~A})$. We also detected the activities of SOD, CAT, and GSH after treatment with CUR. Figure 3B-D show that CUR treatment $(50 \mathrm{mg} / \mathrm{kg}$ and $100 \mathrm{mg} / \mathrm{kg}$ ) effectively reversed the decrease of SOD, CAT, and GSH activity induced by OXA administration $(P<0.05)$. These results indicate that CUR exerts antioxidant effects on mice with OXA-induced liver injury. 

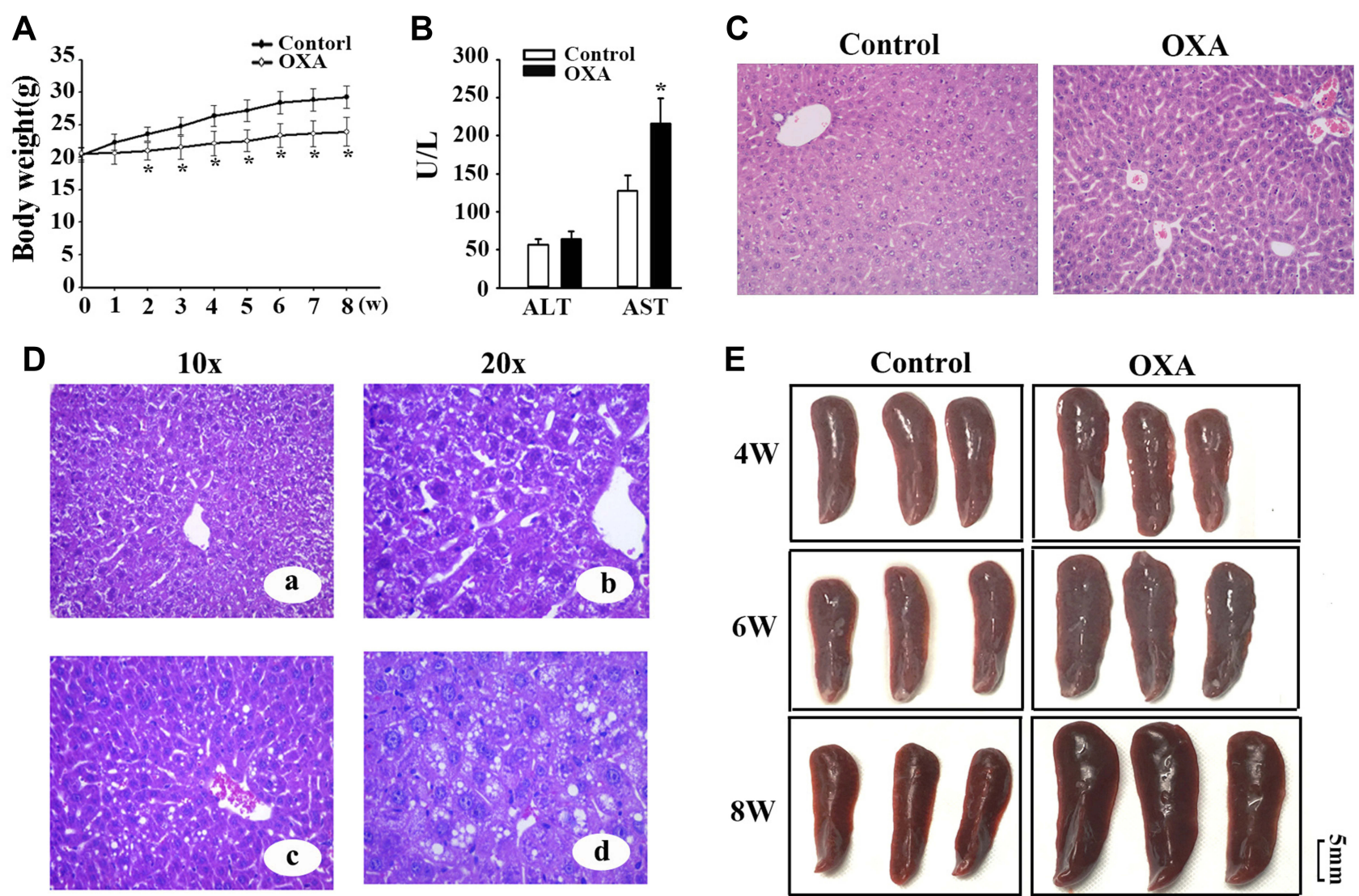

E
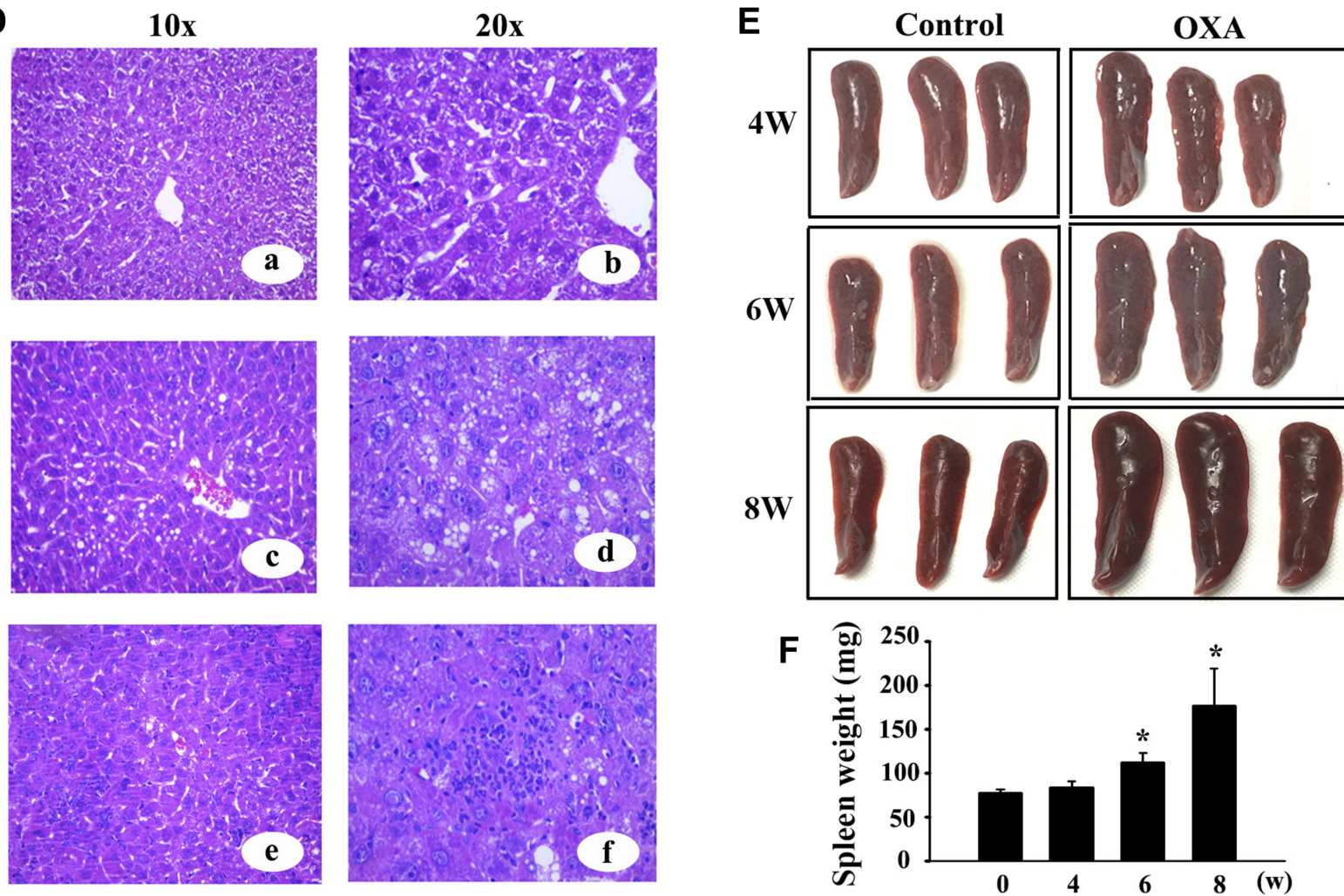

Figure I OXA-induced liver injury and splenomegaly in mice. (A) Time course study of body weight after treatment with i.p. oxaliplatin (OXA). (B) Serum ALT and AST levels one week after the final OXA dose. (C) Histological examination of liver tissues after treatment with OXA for eight weeks (H\&E staining, original magnification: I00x). (D) Sinusoidal dilation $(a, b)$, steatosis (c, d), and inflammatory cell infiltration (e, f) were detected in the liver sections of the OXA-treated group (H\&E staining, original magnification: 200x). (E and F) Images of the spleen (E) and average spleen weights (F) of wild-type mice or those treated with OXA for four, six, and eight weeks. The edge of the spleen had a saw-tooth profile after treatment with OXA for four weeks, splenomegaly and the saw-tooth edge were observed at six weeks, and splenomegaly was detected after eight weeks. The results are shown as the mean \pm SD for each group. $* P<0.05$ vs control group.

\section{CUR Inhibits OXA-Induced Cytokine Response}

OXA-induced liver injury is associated with an increase in the expression of a variety of chemokines/pro-inflammatory cytokines. ${ }^{10}$ In our study, RT-qPCR confirmed the upregulation of chemokines/pro-inflammatory mediators, including CXCL1, CXCL2, and MCP-1 $(P<0.01)$ in liver after OXA exposure, which was reversed by CUR treatment $(50 \mathrm{mg} / \mathrm{kg}$ and $100 \mathrm{mg} / \mathrm{kg}$ ) (Figure 4A-C). These results indicated that CUR inhibits the OXA-induced cytokine response in mice with OXA-induced liver injury.

\section{CUR Reverses the Pro-Thrombotic Environment Within OXA-Injured Hepatic Sinusoids}

Previous studies have demonstrated that OXA-induced liver sinusoids injury is correlated with over activation of the coagulation cascade. ${ }^{10}$ In the present study, RTqPCR analysis revealed that the mRNA levels of vWF, a key component in platelet adhesion, ${ }^{24}$ significantly increased in the livers of the OXA-treated group $(P<0.01)$ (Figure 4D). CUR treatment $(100 \mathrm{mg} / \mathrm{kg})$ significantly inhibited this increase $(P<0.01)$ (Figure 4D). 

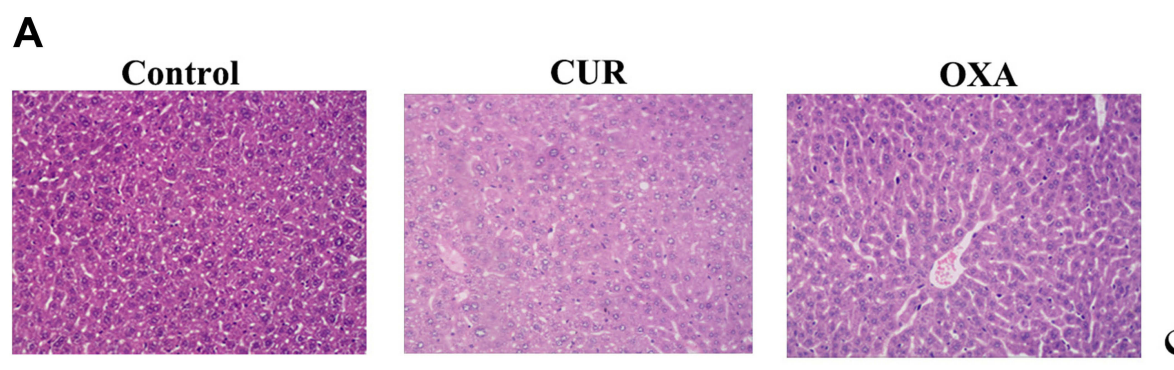

C

OXA+ CUR $50 \mathrm{mg} / \mathrm{kg}$
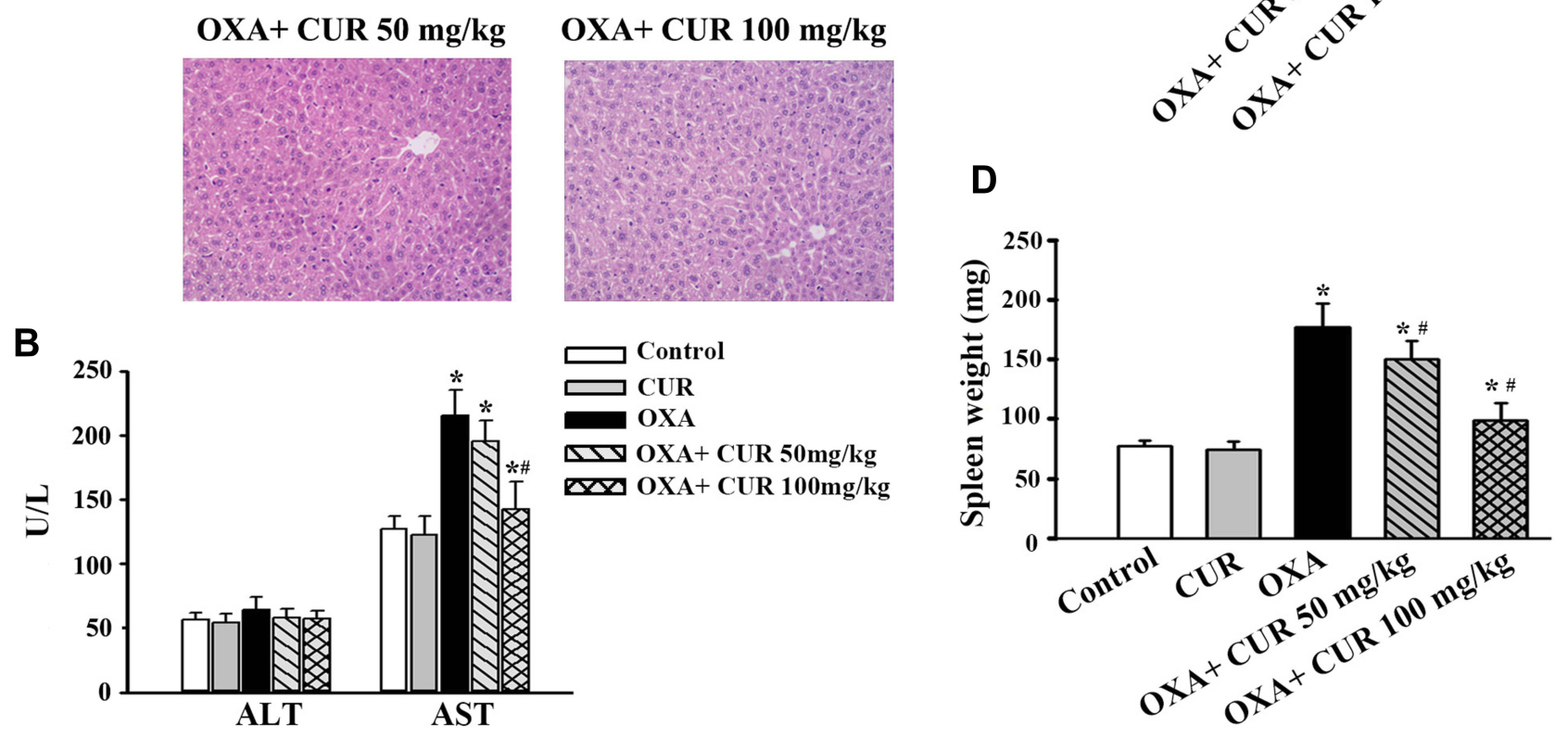

Figure 2 CUR attenuates OXA-induced liver injury and splenomegaly. Mice were randomly classified into five groups: (1) Control group: administered with 5\% glucose; (2) CUR group: treated orally with CUR via gavage; (3) OXA group: treated with OXA on a weekly basis; (4) OXA+CUR $50 \mathrm{mg} / \mathrm{kg}$ group: treated with OXA (as OXA group) plus CUR (50 mg/kg, 30 min before and three consecutive days after every OXA injection); and (5) OXA+CUR $100 \mathrm{mg} / \mathrm{kg}$ group: treated with OXA (as OXA group) plus CUR (100 mg/kg, $30 \mathrm{~min}$ before and three consecutive days after every OXA injection). After treatment for eight weeks, the mice were euthanized one week following the final dose of OXA. (A) Liver histopathology was examined in each group three days after the final OXA dose (H\&E staining, original magnification: I00×). (B) The serum ALT and AST levels of each group were evaluated three days after the final OXA dose. (C) Representative images of the spleens of each group. (D) The spleen weight of each group. The results are presented as the mean \pm standard deviation of five mice from each group. ${ }^{*} P<0.05$ vs control group, ${ }^{\#} P<0.01$ vs $O X A$ group.

Furthermore, OXA was also associated with an increase the hepatic expression of PAI-1, which was notably inhibited by CUR treatment $(50 \mathrm{mg} / \mathrm{kg}$ and $100 \mathrm{mg} / \mathrm{kg})$ $(P<0.01)$ (Figure 4E).

\section{CUR Activates the Nrf2 Pathway in OXA-Induced Liver Injury}

Phase II antioxidant enzymes including HO-1 and NQO1 contribute to the detoxifying step in drug metabolism. To address whether these enzymes are involved in mediating the beneficial effect of CUR, we investigated their expression in the liver. Figure 5A show that HO-1 and NQO1 were downregulated in the OXA treatment group $(P<0.01$ and $P<0.01$ ), whereas CUR treatment (50 and $100 \mathrm{mg} / \mathrm{kg}$ ) reversed the decreased expression of HO-1 and NQO1 $(P<0.01$ and $P<0.01)$. Nrf2, an important transcription factor, has been reported as an upstream regulator of
HO-1 and NQO1. To confirm whether CUR exerts its protective effect through the Nrf2 pathway, we measured the Nrf2 protein expression in the nuclear fraction of liver tissue. Figure 5B shows that Nrf2 protein expression increased in the liver tissues after CUR treatment (50 mg/kg and $100 \mathrm{mg} / \mathrm{kg})(P<0.01)$. Finally, CUR alone significantly increased the expression of HO-1, NQO1, and Nrf2 compared with the control group $(P<0.01)$ (Figure 5A-B).

\section{The CUR-Induced Nrf2 Activation and the Hepatoprotective Effect of CUR Were Abolished by Brusatol}

The Nrf2 inhibitor brusatol was administrated to further confirm CUR-induced Nrf2 activation in mice with OXAinduced liver injury. Figure 6A shows that the $100 \mathrm{mg} / \mathrm{kg}$ 

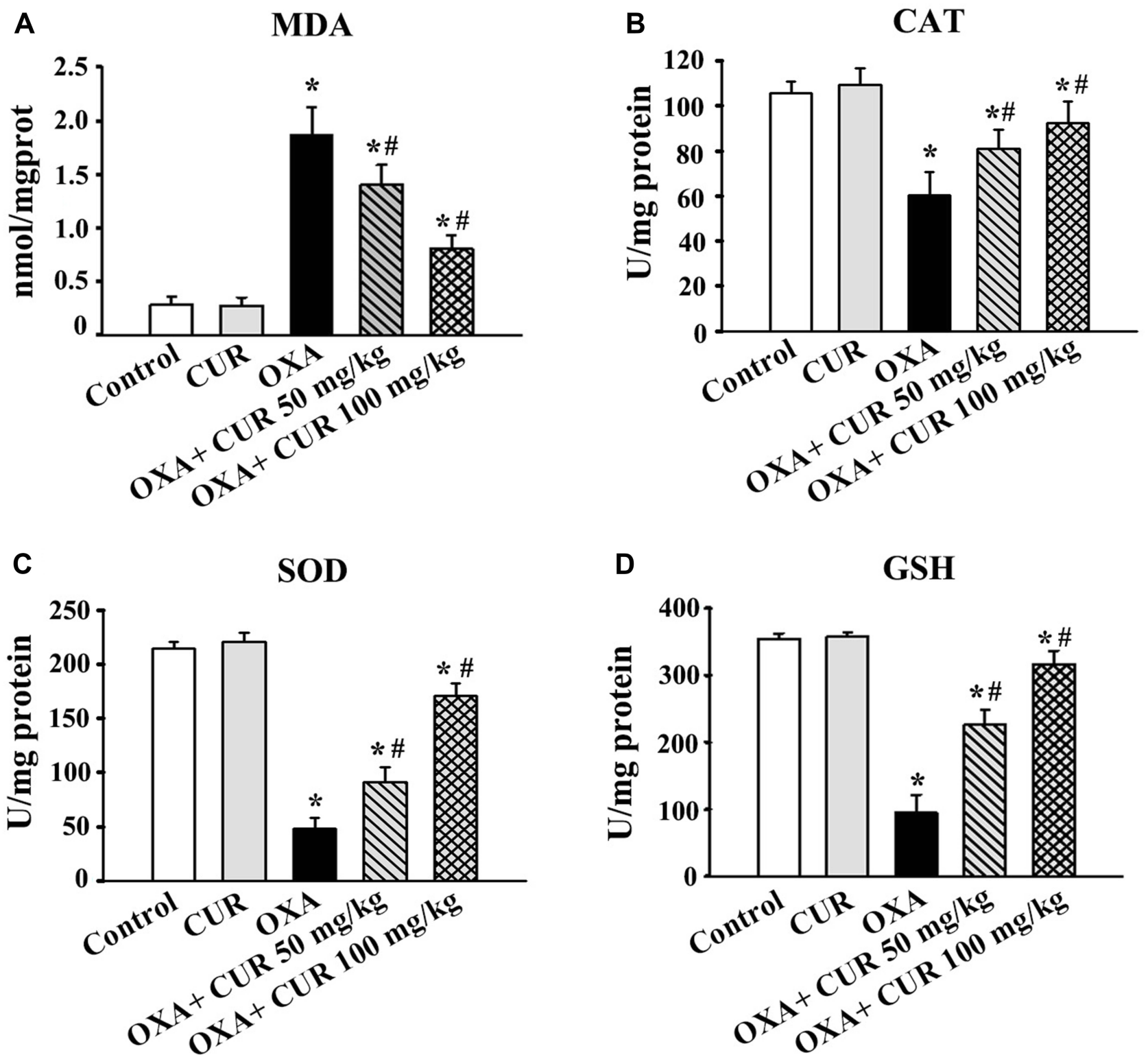

Figure 3 Effects of CUR on OXA-induced oxidative stress. (A) MDA levels and activities of (B) SOD, (C) CAT, and (D) GSH in each group were measured. The results are presented as the mean \pm standard deviation of five mice from each group. ${ }^{* P}<0.01$ vs control group, ${ }^{\#} P<0.01$ vs $O X A$ group.

CUR treatment significantly increased the expression level of Nrf2 $(P<0.05)$ in mice with OXA-induced liver injury, whereas brusatol attenuated this increase. Further, H\&E-stained liver sections revealed more severe pathological sinusoidal injury in the OXA+CUR+Bru group compared to the OXA+CUR group, and the pathology was similar between the OXA+CUR+Bru group and the OXA group (Figure 6B). Moreover, the splenomegaly was more severe in the $\mathrm{OXA}+\mathrm{CUR}+\mathrm{Bru}$ group than in the OXA + CUR group (Figure 6C and D). These results indicated that the Nrf2 activation and the hepatoprotective effect in
OXA-treated mice by CUR treatment were abolished by brusatol.

The Increased Expression of HO-I and NQOI Induced by CUR Was Abolished by Brusatol

Western blot analysis showed that consistent with the decreased activity of Nrf2 after treatment with brusatol, HO-1 ( $\mathrm{P}<0.05$; Figure 7A and $\mathrm{B})$ and NQO1 $(\mathrm{P}<0.05$; Figure $7 \mathrm{~A}$ and $\mathrm{C}$ ) expression in liver tissue was significantly 


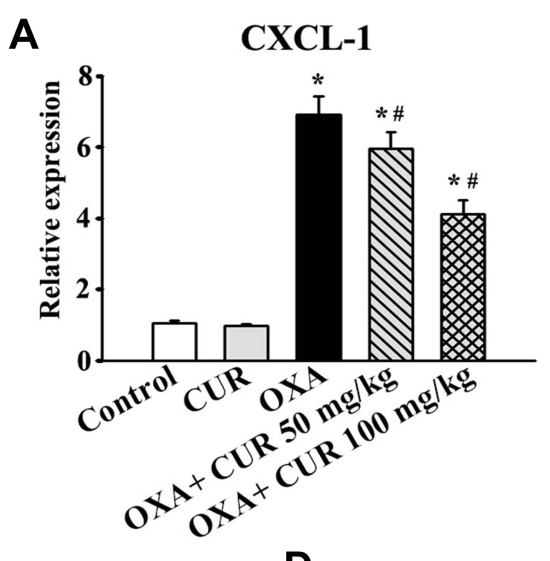

D

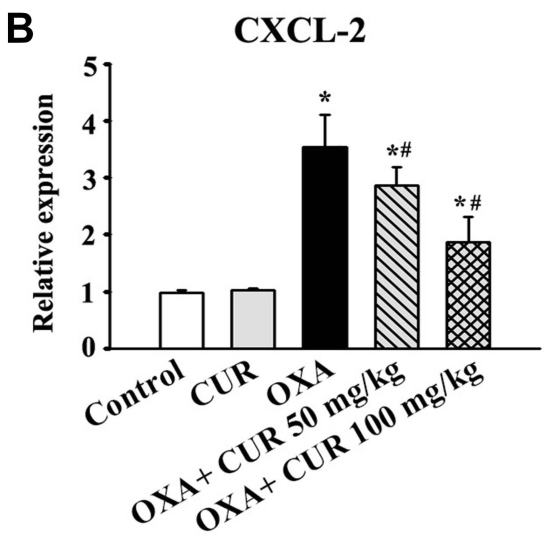

vWF

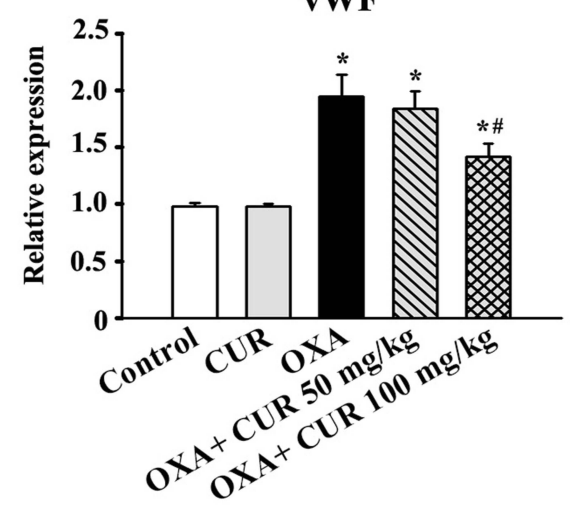

E

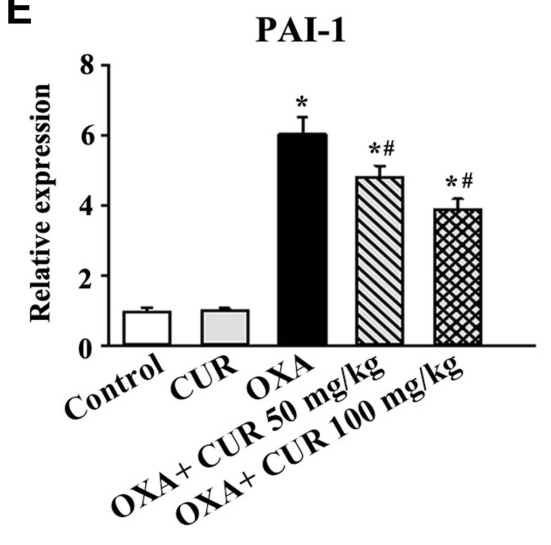

PAI-1

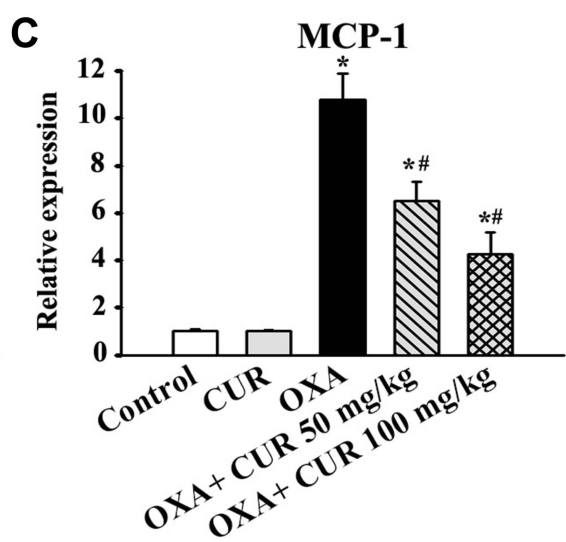

Figure 4 Effects of CUR on OXA-induced cytokine responses and the coagulation cascade. mRNA expression levels of CXCLI (A), CXCL2 (B), MCP-I (C), vWF (D), and PAI-I (E) of each group were measured. The results are presented as the mean \pm standard deviation of five mice from each group. ${ }^{*} P<0.0 \mathrm{I}$ vs control group, ${ }^{\#} P<0.0 \mathrm{I}$ vs OXA group.

decreased in the $\mathrm{OXA}+\mathrm{CUR}+\mathrm{Bru}$ group compared to the OXA+CUR group.

\section{Discussion}

Early studies have shown that OXA hepatotoxicity mainly manifests as hepatic steatosis and sinus injury. ${ }^{4}$ Further research has shown that OXA hepatotoxicity is most likely accompanied by splenomegaly. ${ }^{25-28}$ In addition, the increase in the splenic volume is significantly higher in patients with SOS than in those without SOS. ${ }^{27}$ Prior to this report, animal models have been used to study the hepatotoxic effects of OXA, which were mainly characterized by hepatic injury. In the current study, the histological changes in sinusoidal dilatation, steatosis, and inflammatory cell infiltration were observed in the liver of OXA-treated mice. OXA also induced oxidative stress in the liver of mice, as confirmed by a significant increase in the levels of MDA, as well as a significant decrease in the activity of the antioxidant enzymes CAT, SOD, and GSH. Moreover, the edge of the spleen had a saw-tooth profile that appears during the early stages of OXA treatment. As the number of experiments increased, splenic volume significantly increased, and splenomegaly was observed. To the best of our knowledge, this is the first report of a reproducible experimental chronic model of OXA-induced liver injury with splenomegaly, which can be used to investigate the molecular pathogenesis of this condition.

CUR, a nontoxic component of the turmeric plant Curcuma longa, is a popular phytochemical for the prevention of toxicity in various tissues and cells..$^{1,15,16}$ In this study, we observed that CUR could relieve the OXAinduced liver injury and splenomegaly and additionally attenuate oxidative stress in mice. Our results showed that OXA-induced liver injury, characterized by the pathological sinusoidal injury and serum AST activity, was significantly attenuated by CUR treatment. These results strongly suggest that CUR effectively attenuates OXAinduced liver injury.

There is evidence that oxidative stress is responsible for the hepatotoxicity of OXA. ${ }^{10}$ In this study, the levels of the oxidative indicator MDA increased, whereas the levels of antioxidant enzymes SOD, CAT, and GSH significantly 

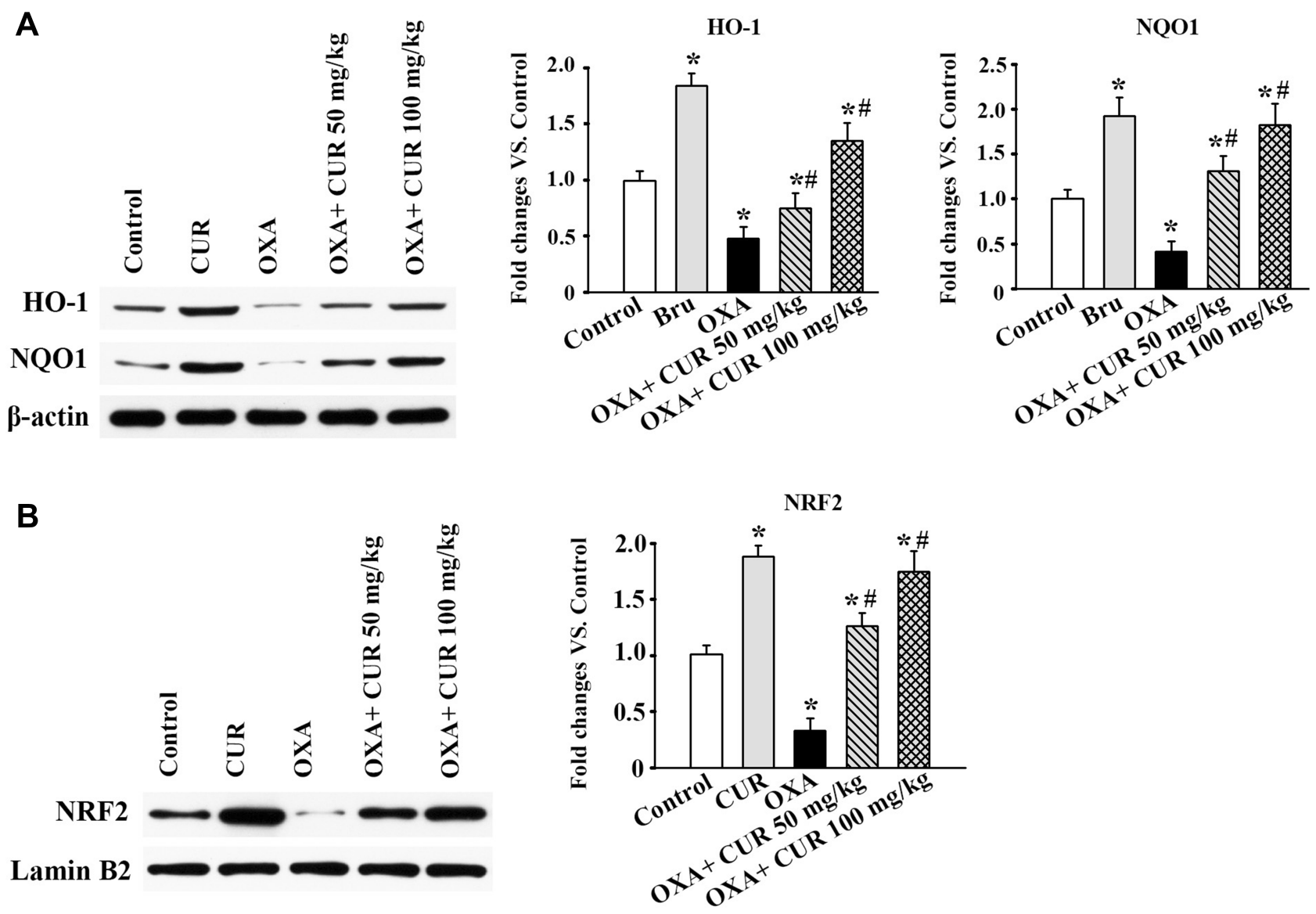

Figure 5 Effects of CUR on the Nrf2 pathway in the liver of mice with OXA-induced liver injury. The protein expression levels of HO-I (A), NQOI(A), and Nrf2 (B) in the liver tissues of each group were measured by Western blotting, and the data are expressed as the fold change vs the control group. The results are presented as the mean \pm standard deviation of five mice from each group. ${ }^{*} P<0.01$ vs control group, ${ }^{\#} P<0.01$ vs OXA group.

decreased after OXA exposure. After CUR treatment, the level of MDA decreased and that of SOD, CAT, and GSH increased. These results suggest that CUR can effectively ameliorate OXA-induced hepatic oxidative stress.

Further investigation suggested that the antioxidant properties of CUR in OXA-induced liver injury is associated with the activation of Nrf2, which induces phase II detoxifying and antioxidant enzymes. Nrf2, a member of the leucine zipper transcription factor family, is regarded as one of the pivotal regulators of cytoprotective and detoxification genes that prevent oxidative stress. ${ }^{15,29}$ Under normal physiological conditions, Nrf2 binds to a Kelch-like ECH-associated protein (Keap1). ${ }^{29}$ After exposure to oxidative stress, Nrf2 dissociates from Keap1 and further upregulates the transcription of multiple antioxidant response element (ARE)controlled genes, ultimately inducing the expression of a variety of antioxidant enzymes and phase II drugmetabolizing enzymes, including HO-1 and NQO1, which suppress oxidative stress. ${ }^{29}$ Activation of the Nrf2 signaling pathway mitigates oxidative stress and the generation of proinflammatory cytokines. ${ }^{15}$ The results of this study showed that hepatic NRF2 protein expression decreased in mice treated with OXA. CUR treatment significantly increased the expression of both hepatic Nrf2 protein and two typically recognized Nrf2 downstream target genes, HO-1 and NQO1. However, when the Nrf2 inhibitor brusatol was added, the effects of CUR on the expression of $\mathrm{Nrf} 2$ and its downstream target genes HO-1 and NQO1 were abolished. Meanwhile, more severe pathological sinusoidal injury and splenomegaly were observed in the $\mathrm{OXA}+\mathrm{CUR}+$ brusatol treated mice than in the OXA+ CUR treatment mice. These findings indicate that CUR, through activation of the Nrf2-ARE pathway, reduces OXA-induced hepatotoxicity and oxidative injuries in vivo.

One of the mechanisms for cellular excretion of OXA is via conjugation of the active drug to GSH, which can then lead to intracellular depletion of this antioxidant. ${ }^{30}$ GSH can resist oxidative stress by serving as a substrate 
A
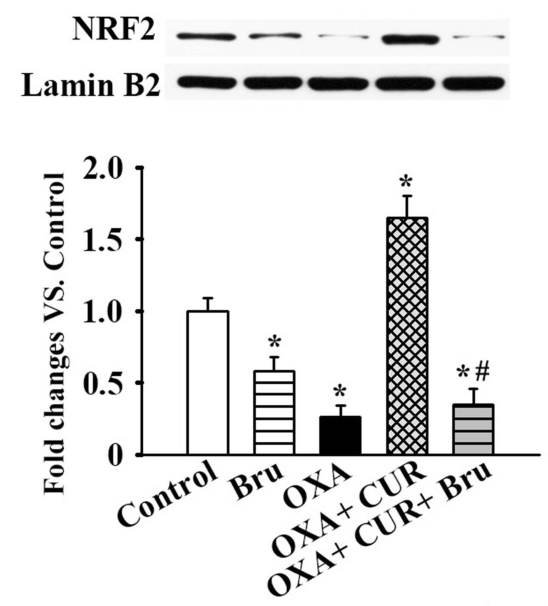

C

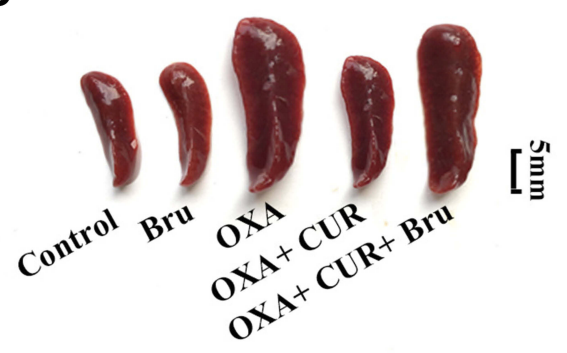

B
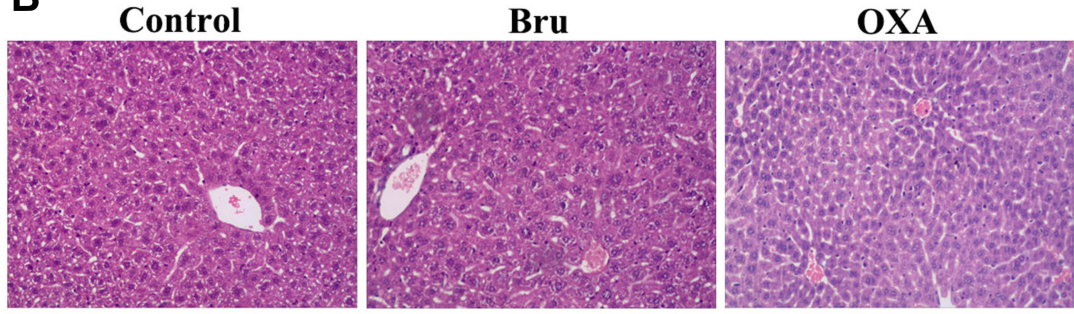

OXA+ CUR

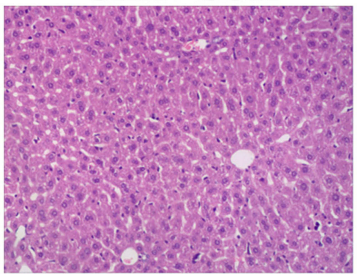

OXA+ CUR + Bru

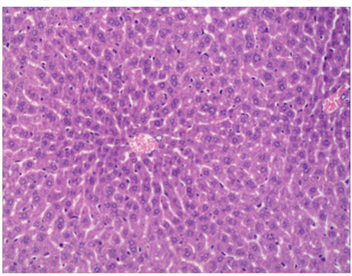

D

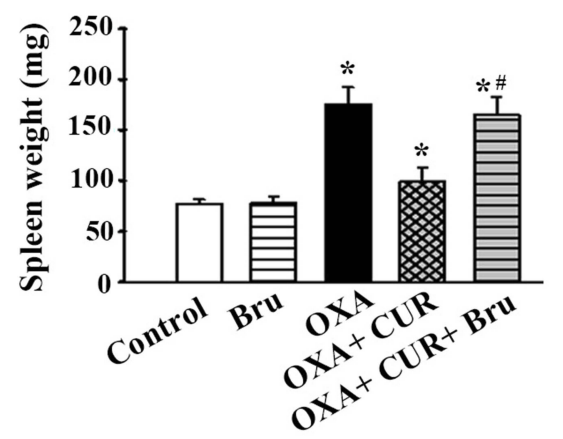

Figure 6 Effects of CUR and brusatol on the Nrf2 in the liver of mice with OXA-induced liver injury. Mice were randomly allocated into five groups: (I) Control group: administered with $5 \%$ glucose; (2) Bru group: administered with brusatol; (3) OXA group: treated with OXA on a weekly basis; (4) OXA+CUR: treated with OXA (as OXA group) plus CUR (100 mg/kg, 30 min before, and three consecutive days after, every OXA injection); (5) OXA+ CUR+ Bru: treated with OXA and CUR (as OXA+CUR group) plus brusatol ( $2 \mu \mathrm{mol} \mathrm{kg}-\mathrm{I}, 2 \mathrm{~h}$ before each CUR gavage). (A) The protein expression levels of Nrf2 in liver tissues of each group were measured by Western blotting. The results are presented as the mean \pm standard deviation of five mice from each group. (B) Liver histopathology was examined in each group three days after the final OXA dose (H\&E staining, original magnification: 100x). (C) Representative images of the spleens of each group. (D) The spleen weight of each group. The results are presented as the mean \pm standard deviation of five mice from each group. ${ }^{*} P<0.05$ vs control group, ${ }^{\#} P<0.01$ vs $O X A+C U R$ group.

A

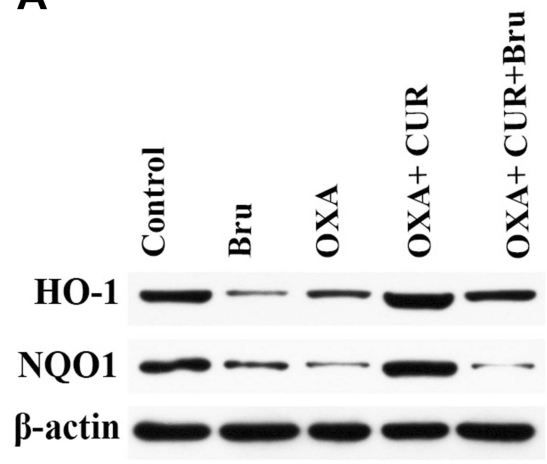

B

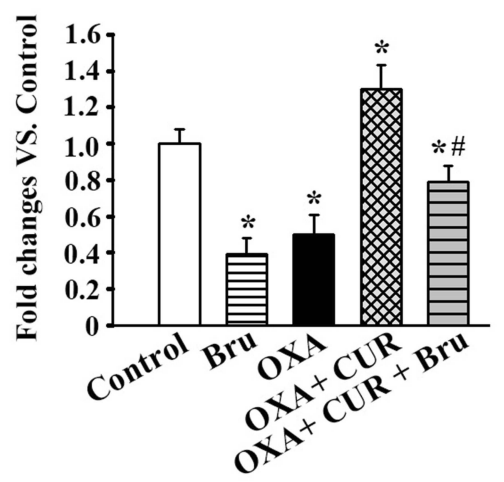

C

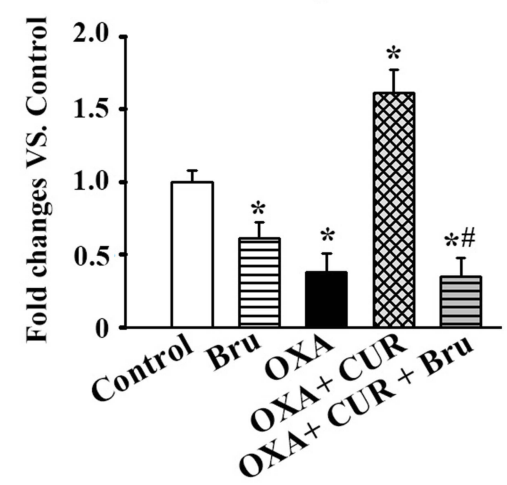

Figure 7 Effects of CUR and brusatol on the expression of HO-I and NQOI in the liver of OXA-treated mice. (A) The protein expression levels of HO-I and NQOI in the liver tissue of each group were measured by Western blotting. $\mathrm{HO}-\mathrm{I}(\mathbf{B})$ and $\mathrm{NQOI}(\mathrm{C})$ bands of each group were scanned, and the data are expressed as the fold change vs the control group. The results are presented as the mean \pm standard deviation of five mice from each group. ${ }^{*}<0.01$ vs control group, ${ }^{*} P<0.01$ vs $O X A+C U R$ group. 
for antioxidative enzymes, including GSH-Px, which converts hydroperoxide into less harmful fatty acids, water, and GSH disulfide. ${ }^{31}$ Our results indicated that the disturbance of GSH balance, i.e., the reduction hepatic GSH levels by OXA exposure, was reversed by CUR treatment. CUR treatment increased levels of GSH, which further inhibit the oxidative stress response induced by OXA. In addition, activation of Nrf2 could enhance antioxidant GSH synthesis. ${ }^{29,32} \mathrm{GSH}$, attaining concentrations in the high millimolar range in the liver, can resist oxidative stress by serving as a substrate for antioxidative enzymes, converting hydroperoxide into less harmful fatty acids, water, and GSH disulfide. ${ }^{33} \mathrm{Nrf} 2$ could elevate the expression of the gene coding for the rate-limiting enzyme in glutathione synthesis and glutamate cysteine ligase (GCL). ${ }^{34}$ Through the PI3K/Akt-dependent pathway, Nrf2 could increase GSH synthesis. ${ }^{32}$ OXA treatment can lead to intracellular depletion of GSH. ${ }^{30}$ Our previous research has shown that GSH supplementation effectively alleviates OXA-induced hepatic oxidative stress in mice. ${ }^{12}$ The results of this study revealed that CUR treatment, possibly through the Nrf2 pathway, significantly increases GSH levels, thus further inhibiting the oxidative stress response induced by OXA.

CUR has established anti-inflammatory properties, and is known to protect cells against hyperactivated immune system via diverse pathways. ${ }^{21}$ CUR is a known inhibitor of NF- $\kappa$ B, a key regulator of inflammation, which inhibits the expression of interleukins, including IL-1, IL-2, and IL-6, as well as TNF- $\alpha$, CXCL1, and CXCL8. ${ }^{15,21}$ Our results indicated that CUR treatment mitigated OXAinduced hepatocellular injury by inhibiting the production of the pro-inflammatory cytokines CXCL1, CXCL2, and MCP-1. Therefore, in addition to the inhibition of oxidative stress, the protective effect of CUR also correlated with the inhibition of hepatic inflammatory.

Splenomegaly is one of the clinically important OXA adverse effects that has been characterized. ${ }^{25-27}$ OXA has been found to induce disruption of the sinusoidal endothelium and collagen deposition in the perisinusoidal space, which result in portal hypertension, splenomegaly, and, finally, thrombocytopenia. ${ }^{9}$ Meanwhile, splenomegaly and thrombocytopenia were useful indicators of the presence of OXA-based chemotherapy-associated liver injury. ${ }^{28}$ A previous study has also shown that an increase in spleen volume possibly precedes a significant peripheral neuropathy that could be a potential marker for OXA-induced toxicity. ${ }^{25}$ In this study, we found that CUR treatment not only relieved
OXA-induced liver injury, but also attenuated OXA-induced splenomegaly. This result further proves that CUR can alleviate the OXA-induced hepatic toxicity in vivo. To the best of our knowledge, this study is the first report of a medication to treat or prevent OXA-induced splenomegaly in an animal model.

Our study also observed the effects of CUR on the coagulation cascade. More platelets were observed in contact with hepatocytes and with hepatocyte destruction in patients with severe hepatic sinusoidal injury (HSI) after OXA chemotherapy than in those with mild HIS. ${ }^{9}$ The disruption of sinusoidal endothelial cells by OXA allows activated platelets to migrate into the space of Disse and aggregate. ${ }^{9}$ These activated platelets secrete various growth factors, including PAI-1, platelet-activating factor (PAF), thromboxane (TX) A2, thrombospondin, and vascular endothelial growth factor (VEGF)-A, which may cause liver injury. ${ }^{35}$ Indeed, hepatic levels of vWF, PAI-1, PAR1, PAR2, TXA2, and VEGF-A are known to be upregulated after OXA treatment. ${ }^{9,10,13}$ The present results demonstrated that CUR treatment reverses the pro-thrombotic environment within OXA-injured hepatic sinusoids, which was confirmed by the results that increased levels of vWF and PAI-1 induced by OXA were inhibited after CUR treatment. OXA treatment also caused an increase in hepatic expression of the PAI-1, which was inhibited notably by CUR treatment.

Despite its beneficial effects, the limited bioavailability of curcumin is highlighted as a major concern and restricts its application in the body. ${ }^{36}$ Yet, numerous approaches have been undertaken to improve the bioavailability of curcumin, including nanoparticles, liposomes, and defined phospholipid complexes. There is increasing evidence that enhanced bioavailability of curcumin through these new approaches exert better effects on inflammation and oxidative stress compared to conventional curcumin. ${ }^{37,38}$ Therefore, using new novel formulations of curcumin, such as nanoparticles ${ }^{37}$ and liposomes, ${ }^{38}$ may more effectively attenuate OXA-induced liver injury and oxidative stress compared to the conventional curcumin which we used in these study. This warrants further investigation.

Extensive research within the last decade involving cell cultures and in rodents has revealed the anti-tumor effect of CUR, both as a single compound and in combination with chemotherapeutic agents. ${ }^{15,16}$ CUR can sensitize tumor cells to different chemotherapeutic agents, including OXA. ${ }^{15}$ A recent trial showed that CUR is a safe and tolerable adjunct to FOLFOX (5-fluorouracil, folinic acid, and oxaliplatin) chemotherapy in patient-derived colorectal liver metastases. ${ }^{39}$ 
The addition of CUR as an adjunct to oxaliplatin enhances OXA toxicity in malignant cells, which potentially allows an OXA dose reduction and decrease of its adverse effects. ${ }^{15}$ Whether the hepatoprotective effect of CUR observed in the current study may contribute to the anticancer effects of OXAbased chemotherapy needs further study.

\section{Conclusions}

We described a novel, reproducible model of liver injury and splenomegaly following OXA chemotherapy. We used this to elucidate the protective effects of CUR against OXA-induced hepatotoxicity. We demonstrated that CUR effectively attenuated OXA-induced liver injury and splenomegaly in vivo. The protective effect of CUR on hepatotoxicity injuries was correlated with the attenuation of oxidative stress, inflammation, and the coagulation pathway. Additionally, this study provided mechanistic insights into the protective roles of CUR, in which it could effectively upregulate the expression of transcription factor $\mathrm{Nrf} 2$, as well as Nrf2 downstream targets, thus mitigating OXA-induced oxidative stress. Our study may therefore suggest the use of a potential chemopreventive dietary component to counteract the toxicant-induced liver injury in clinical practice.

\section{Ethical Statement}

All animal studies were performed according to the guidelines of the Chinese Council on Animal Care and were approved by the Guangxi Medical University Cancer Hospital Committees on Animal Experimentation (LW2019033; Nanning, China).

\section{Author Contributions}

All authors contributed to data analysis, drafting or revising the article, gave final approval of the version to be published, and agree to be accountable for all aspects of the work.

\section{Funding}

The present study was partially supported by the SelfRaised Funds of Guangxi Health Department (Grant no. Z2016483), the Guangxi Natural Science Foundation (Grant no. 2016GXNSFBA380218; 2019JJA140558), the Guangxi Basic Ability Promotion Project of Middle-aged and Young Teachers in Colleges and Universities (Grant no. 2017KY0121), and the Guangxi Key Laboratory of Molecular Medicine in Liver Injury and Repair (Grant no.16-140-46-18).

\section{Disclosure}

The authors report no conflicts of interest in this work.

\section{References}

1. Bai X, Chen Y, Hou X, Huang M, Jin J. Emerging role of NRF2 in chemoresistance by regulating drug-metabolizing enzymes and efflux transporters. Drug Metab Rev. 2016;48(4):541-567. doi:10.1080/ 03602532.2016.1197239

2. Riddell IA. Cisplatin and oxaliplatin: our current understanding of their actions. Met Ions Life Sci. 2018;18. doi:10.1515/ 9783110470734-007

3. Jardim DL, Rodrigues CA, Novis YA, Rocha VG, Hoff PM. Oxaliplatin-related thrombocytopenia. Ann Oncol. 2012;23 (8):1937-1942. doi:10.1093/annonc/mds074

4. Rubbia-Brandt L, Lauwers GY, Wang H, et al. Sinusoidal obstruction syndrome and nodular regenerative hyperplasia are frequent oxaliplatin-associated liver lesions and partially prevented by bevacizumab in patients with hepatic colorectal metastasis. Histopathology. 2010;56(4):430-439. doi:10.1111/j.1365-2559.2010.03511.x

5. Rubbia-Brandt L, Audard V, Sartoretti P, et al. Severe hepatic sinusoidal obstruction associated with oxaliplatin-based chemotherapy in patients with metastatic colorectal cancer. Ann Oncol. 2004;15 (3):460-466. doi:10.1093/annonc/mdh095

6. Vauthey JN, Pawlik TM, Ribero D, et al. Chemotherapy regimen predicts steatohepatitis and an increase in 90-day mortality after surgery for hepatic colorectal metastases. J Clin Oncol. 2006;24 (13):2065-2072. doi:10.1200/JCO.2005.05.3074

7. Nakano H, Oussoultzoglou E, Rosso E, et al. Sinusoidal injury increases morbidity after major hepatectomy in patients with colorectal liver metastases receiving preoperative chemotherapy. Ann Surg. 2008;247(1):118-124. doi:10.1097/SLA.0b013e31815774de

8. Tamandl D, Klinger M, Eipeldauer S, et al. Sinusoidal obstruction syndrome impairs long-term outcome of colorectal liver metastases treated with resection after neoadjuvant chemotherapy. Ann Surg Oncol. 2011;18(2):421-430. doi:10.1245/s10434-010-1317-4

9. Tajima H, Ohta T, Miyashita T, et al. Oxaliplatin-based chemotherapy induces extravasated platelet aggregation in the liver. $\mathrm{Mol}$ Clin Oncol. 2015;3(3):555-558. doi:10.3892/mco.2015.512

10. Robinson SM, Mann J, Vasilaki A, et al. Pathogenesis of FOLFOX induced sinusoidal obstruction syndrome in a murine chemotherapy model. J Hepatol. 2013;59(2):318-326. doi:10.1016/j.jhep.2013.04.014

11. de Andrade KQ, Moura FA, dos Santos JM, de Araujo OR, de Farias Santos JC, Goulart MO. Oxidative stress and inflammation in hepatic diseases: therapeutic possibilities of N-Acetylcysteine. Int J Mol Sci. 2015;16(12):30269-30308. doi:10.3390/ijms161226225

12. Lin Y, Li Y, Hu X, et al. The hepatoprotective role of reduced glutathione and its underlying mechanism in oxaliplatin-induced acute liver injury. Oncol Lett. 2018;15(2):2266-2272. doi:10.3892/ol.2017.7594

13. Zou X, Wang Y, Peng C, et al. Magnesium isoglycyrrhizinate has hepatoprotective effects in an oxaliplatininduced model of liver injury. Int J Mol Med. 2018;42(4):2020-2030. doi:10.3892/ijmm.2018.3787

14. Schwingel TE, Klein CP, Nicoletti NF, et al. Effects of the compounds resveratrol, rutin, quercetin, and quercetin nanoemulsion on oxaliplatin-induced hepatotoxicity and neurotoxicity in mice. Naunyn Schmiedebergs Arch Pharmacol. 2014;387(9):837-848. doi:10.1007/ s00210-014-0994-0

15. Zangui M, Atkin SL, Majeed M, Sahebkar A. Current evidence and future perspectives for curcumin and its analogues as promising adjuncts to oxaliplatin: state-of-the-art. Pharmacol Res. 2019;141:343-356. doi:10.1016/j.phrs.2019.01.020

16. Goel A, Aggarwal BB. Curcumin, the golden spice from Indian saffron, is a chemosensitizer and radiosensitizer for tumors and chemoprotector and radioprotector for normal organs. Nutr Cancer. 2010;62(7):919-930. doi:10.1080/01635581.2010.509835 
17. Lee HS, Li L, Kim HK, et al. The protective effects of Curcuma longa Linn. extract on carbon tetrachloride-induced hepatotoxicity in rats via upregulation of Nrf2. J Microbiol Biotechnol. 2010;20 (9):1331-1338. doi:10.4014/jmb.1002.03010

18. Yu C, Mei XT, Zheng YP, Xu DH. Zn(II)-curcumin protects against hemorheological alterations, oxidative stress and liver injury in a rat model of acute alcoholism. Environ Toxicol Pharmacol. 2014;37 (2):729-737. doi:10.1016/j.etap.2014.02.011

19. Qiu P, Sun J, Man S, et al. Curcumin attenuates N-nitrosodiethylamineinduced liver injury in mice by utilizing the method of metabonomics. J Agric Food Chem . 2017;65(9):2000-2007. doi:10.1021/acs.jafc.6b04797

20. Gao S, Duan X, Wang X, et al. Curcumin attenuates arsenic-induced hepatic injuries and oxidative stress in experimental mice through activation of Nrf2 pathway, promotion of arsenic methylation and urinary excretion. Food Chem Toxicol. 2013;59:739-747. doi:10.1016/j.fct.2013.07.032

21. Cavaleri F. Presenting a new standard drug model for turmeric and its prized extract, curcumin. Int J Inflam. 2018;2018:5023429. doi:10.1155/2018/5023429

22. Luo DD, Chen JF, Liu JJ, et al. Tetrahydrocurcumin and octahydrocurcumin, the primary and final hydrogenated metabolites of curcumin, possess superior hepatic-protective effect against acetaminophen-induced liver injury: role of CYP2E1 and Keap1-Nrf2 pathway. Food Chem Toxicol. 2019;123:349-362. doi:10.1016/j.fct.2018.11.012

23. Said Salem NI, Noshy MM, Said AA. Modulatory effect of curcumin against genotoxicity and oxidative stress induced by cisplatin and methotrexate in male mice. Food Chem Toxicol. 2017;105:370-376. doi:10.1016/j.fct.2017.04.007

24. Chen J, Chung DW. Inflammation, von Willebrand factor, and ADAMTS13. Blood. 2018;132(2):141-147. doi:10.1182/blood2018-02-769000

25. El Chediak A, Haydar AA, Hakim A, et al. Increase in spleen volume as a predictor of oxaliplatin toxicity. Ther Clin Risk Manag. 2018;14:653-657. doi:10.2147/TCRM

26. Kang J, Park JS, Ahn SG, et al. Protective effect of Korean red ginseng on oxaliplatin-mediated splenomegaly in colon cancer. Ann Surg Treat Res. 2018;95(3):161-167. doi:10.4174/astr.2018.95.3.161

27. Imai K, Emi Y, Iyama KI, et al. Splenic volume may be a useful indicator of the protective effect of bevacizumab against oxaliplatin-induced hepatic sinusoidal obstruction syndrome. Eur J Surg Oncol. 2014;40(5):559-566. doi:10.1016/j.ejso.2013.12.009

28. Overman MJ, Maru DM, Charnsangavej C, et al. Oxaliplatinmediated increase in spleen size as a biomarker for the development of hepatic sinusoidal injury. J Clin Oncol. 2010;28(15):2549-2555. doi:10.1200/JCO.2009.27.5701
29. Jadeja RN, Upadhyay KK, Devkar RV, Khurana S. Naturally occurring Nrf2 activators: potential in treatment of liver injury. Oxid Med Cell Longev. 2016;2016:3453926. doi:10.1155/2016/3453926

30. Kweekel DM, Gelderblom H, Guchelaar HJ. Pharmacology of oxaliplatin and the use of pharmacogenomics to individualize therapy. Cancer Treat Rev. 2005;31(2):90-105. doi:10.1016/j.ctrv.2004.12.006

31. Buldak RJ, Buldak L, Kukla M, Gabriel A, Zwirska-Korczala K. Significance of selected antioxidant enzymes in cancer cell progression. Pol J Pathol. 2014;65(3):167-175. doi:10.5114/ pjp. 2014.45779

32. Chen Z, Ma X, Zhu Y, et al. Paeoniflorin ameliorates ANIT-induced cholestasis by activating Nrf2 through an PI3K/Akt-dependent pathway in rats. Phytother Res. 2015;29(11):1768-1775. doi:10.1002/ptr.v29.11

33. Yuan L, Kaplowitz N. Glutathione in liver diseases and hepatotoxicity. Mol Aspects Med. 2009;30(1-2):29-41. doi:10.1016/ j.mam.2008.08.003

34. Fu Y, Zheng S, Lin J, Ryerse J, Chen A. Curcumin protects the rat liver from $\mathrm{CCl}$-caused injury and fibrogenesis by attenuating oxidative stress and suppressing inflammation. Mol Pharmacol. 2008;73 (2):399-409. doi:10.1124/mol.107.039818

35. Battinelli EM, Markens BA, Italiano JE Jr. Release of angiogenesis regulatory proteins from platelet alpha granules: modulation of physiologic and pathologic angiogenesis. Blood. 2011;118 (5):1359-1369. doi:10.1182/blood-2011-02-334524

36. Anand P, Kunnumakkara AB, Newman RA, Aggarwal BB. Bioavailability of curcumin: problems and promises. Mol Pharm. 2007;4(6):807-818. doi:10.1021/mp700113r

37. Boarescu PM, Chirila I, Bulboaca AE, et al. Effects of curcumin nanoparticles in isoproterenol-induced myocardial infarction. Oxid Med Cell Longev. 2019;2019:7847142. doi:10.1155/2019/7847142. eCollection 2019.

38. Mohajeri M, Sadeghizadeh M, Najafi F, Javan M. Polymerized nano-curcumin attenuates neurological symptoms in EAE model of multiple sclerosis through down regulation of inflammatory and oxidative processes and enhancing neuroprotection and myelin repair. Neuropharmacology. 2015;99:156-167. doi:10.1016/j.neuropharm.2015.07.013

39. Jalili-Nik M, Soltani A, Moussavi S, et al. Current status and future prospective of Curcumin as a potential therapeutic agent in the treatment of colorectal cancer. $J$ Cell Physiol. 2018;233 (9):6337-6345. doi:10.1002/jcp.26368

\section{Publish your work in this journal}

Drug Design, Development and Therapy is an international, peerreviewed open-access journal that spans the spectrum of drug design and development through to clinical applications. Clinical outcomes, patient safety, and programs for the development and effective, safe, and sustained use of medicines are a feature of the journal, which has also been accepted for indexing on PubMed Central. The manuscript management system is completely online and includes a very quick and fair peer-review system, which is all easy to use. Visit http://www. dovepress.com/testimonials.php to read real quotes from published authors. 